\title{
Why most pre-published research findings are false.
}

\author{
Sebastian Lobentanzer
}

\begin{abstract}
Caused by the recent surge in preprint volume, particularly in the light of the immense rapidity of Covid-19 research, the question arises, "How reliable are the findings that are reported via preprint?" This question poses serious challenges in estimation and validation of the extent of false or even fraudulent science on preprint servers, and has far-reaching implications for editorial policies. As preprint volume continuously grows, but the interval between preprint and publication does not, the limit of peer-review is fast approaching. The scientific merit or validity of preprints is not assessed by preprint service providers, and hence it is feasible to assume that, comparatively, preprints will be less reproducible than peer-reviewed articles. Publication metadata predict a saturation of the peer-review process in the coming decade, and necessitate an open discussion about editorial policies and publication infrastructure in the biomedical field.
\end{abstract}

Introduction: Preprints are rising fast in popularity in the biomedical sciences. This article does not want to judge on the merits of preprint publishing in general; nor does it want to weigh the pros and cons. However, particularly in the context of the developments emerging in the SARSCoV-2 pandemic, the dizzying speed of scientific publications should be seen with caution. This is all the more relevant when research is utilised in policymaking, and especially so if there are ties between researchers, politicians, and society, which is increasingly common due to social media dynamics.

Methods: I deduced from the commonly accepted premise that "most published research findings are false" that, consequently, also preprint findings must in the majority be false.

To assess the landscape of preprint and peer-review publishing and their relationships in the biomedical fields, I analysed metadata collected by Europe PMC ${ }^{2}$ and rxivist.org ${ }^{3}$. Data were downloaded through their respective APIs, R/europepmc and the docker postgreSQL dump of rxivist.org, on April 30 ${ }^{\text {th }}, 2020$. Downloaded data included numbers of preprint and peer-reviewed manuscripts (from Europe PMC, preprints collected from a variety of popular preprint servers), and more detailed data on monthly preprint publications from bioRxiv.org and the interval between their preprint upload and eventual peer-reviewed publication, where applicable (from rxivist.org).

Results: Preprints in the biomedical field are rising in popularity in linear fashion since 2012 (Figure 1A), fast approaching the number of peer-reviewed manuscripts published per year (from hereon out simply called "published" for brevity). Simple extrapolation via linear regression estimates the number of biomedical preprints and published manuscripts to be equal approximately in 2025 at the latest (Supplementary Figure 1). Publication rate, the fraction of bioRxiv preprints that are eventually published, has been steady in the years since their establishment, around 70\% (Figure 1B).

Monthly bioRxiv preprint numbers retrace the general preprint publication trend of other servers, showing a linear increase, and the number of eventually published manuscripts parallels the 
preprint numbers up until the beginning of 2017 (Figure 1C). However, from thereon out, numbers of eventually published manuscripts appear to drop off in comparison, until they reach the plateau of manuscripts too recent to quantitatively be published. An overview of publication intervals (from preprint upload date to publication date) indicates a very consistent mean publication time of $226 \pm_{\text {SD }} 154$ days (Figure 1D, mean from 2014 to 2018). The median publication interval is predictably decreased by the influence of the maximally possible publication interval, which is visible in 2019, and obvious in 2020.

Discussion: Since most published (i.e., peer-reviewed) research findings are false, ${ }^{1}$ it is highly unlikely that, given the published findings being nearly a subset (70\%) of the preprint manuscripts, the proportion of preprint manuscripts with non-false findings is higher than the respective proportion in manuscripts that have passed peer-review. This reasoning is limited to relatively recent times, where the premise of "peer-reviewed articles are a smaller subset of all preprints" holds, and excludes articles that have never been preprints. However, as preprints gain in popularity, the numbers of these never-preprinted articles is bound to decrease.

However, an important question tied to the increase in preprint volume is the saturation of available peer-review capacities. The prediction of 2025 as equilibrium time is limited by the simplistic linear regression, an asymptotic approach of preprint numbers is more probable. However, once preprint numbers in fact equal or even exceed the number of currently peerreviewed manuscripts, the traditional balance of scientific evidence, which is represented by the gold standard peer-review, may shift. The societal consequences of these dynamics are unclear.

In the recent years up until 2017,30\% of preprints did not "make the cut," for whichever reasons that may have been. The developments outlined in Figures 1B\&C allow the conclusion that this percentage of peer-review "dropout" will in all likelihood increase rather than decrease.

Intriguingly, while the publication rate itself stagnates from 2014 to 2017, the distribution becomes much narrower, indicating a growing consistency of publication rate. While the plateau effect visible in Figure 1C can be explained for 2019 onwards by the fact that some preprints have not yet reached the maximally available publication interval, the decrease across 2017 and 2018 may already indicate a saturation effect. The publication interval analysis (Figure 1D) indicates an almost completed cycle for 2017 and 2018, which is inconsistent with the apparent decrease in publication rate visible in Figures $1 \mathrm{~B} \& \mathrm{C}$.

While preprint numbers rise, the publication interval remains stable at seven-and-a-half months, indicating no apparent change in editorial quality or efficiency of the peer-review process itself. Inference about the publication rate is less clear. Lower publication rate in 2018 (Figure 1B) may already indicate a beginning saturation. The most likely result of this saturation will be more stringent editorial practices, particularly regarding the initial screen of manuscripts. Initial submissions are judged less on scientific rigour and rather on factors more relevant to the journal: scientific niche (scope), the potential impact and expected citations of the manuscript, the esteem of authors, and various trends in science. Additionally, due to the fast-acting social media distribution of preprint results, the control function of peer-review may be continuously eroded. The damage that can be done by a faulty preprint in seven months' time is potentially immeasurable. 
In summary, the dynamics surrounding preprints will shape the biomedical publication landscape in the coming decade, and the outcomes will depend on societal and technological developments in publication infrastructure.

Declaration: The author has no conflict of interest to declare.

\section{Bibliography}

1. Ioannidis, J. P. A. Why most published research findings are false. PLoS Med. 2, e124 (2005).

2. The Europe PMC Consortium. Europe PMC: a full-text literature database for the life sciences and platform for innovation. Nucleic Acids Res. 43, D1042-D1048 (2015).

3. Abdill, R. J. \& Blekhman, R. Tracking the popularity and outcomes of all bioRxiv preprints. Elife 8, (2019). 

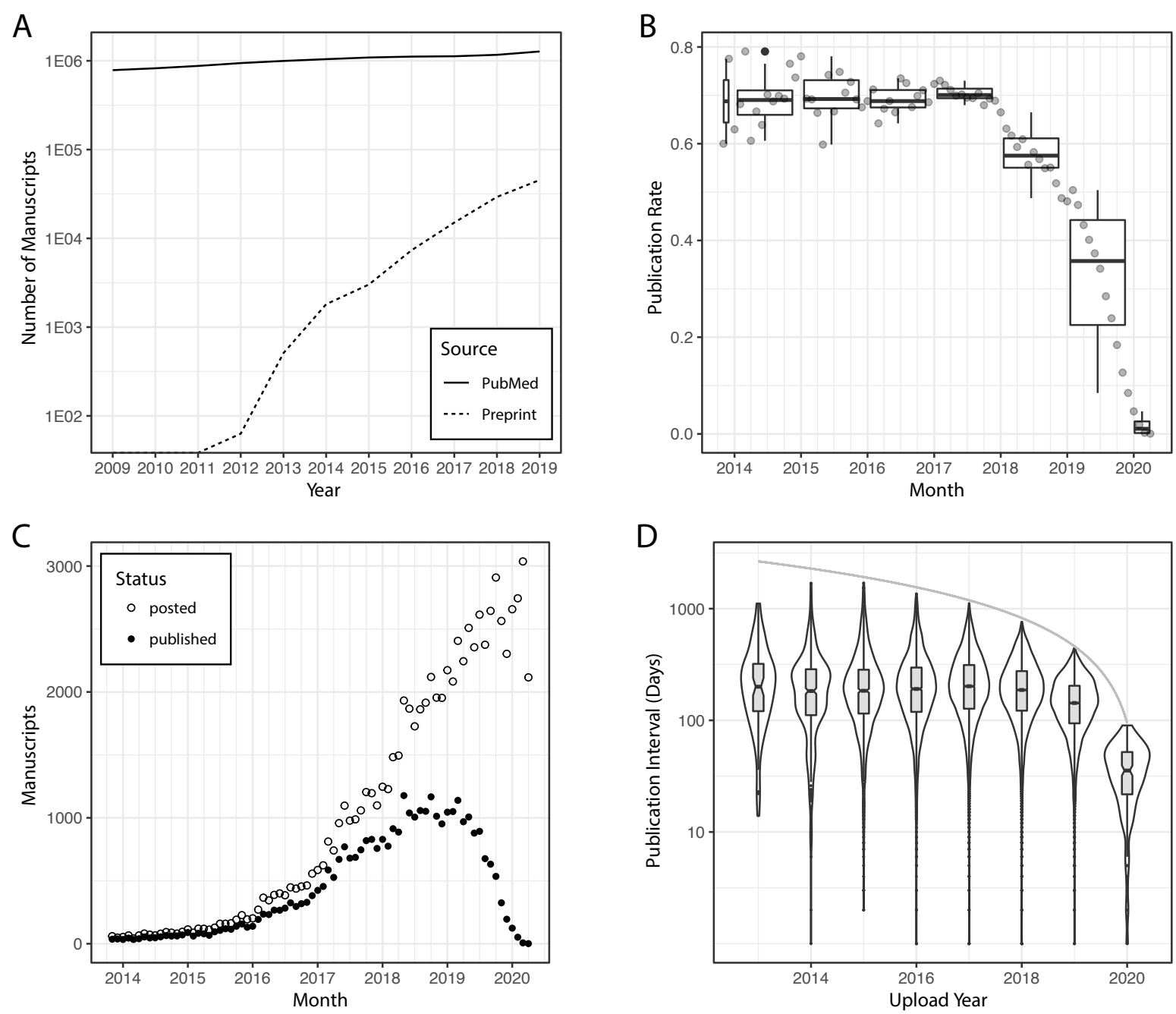

Figure 1: Preprint numbers, publication rate, and time to publication. A) The number of published peer-reviewed manuscripts (indexed by PubMed, solid line) was stable at 1 million per year, while the number of preprints (curated by Europe PMC, dotted line) rose almost linearly from 2012 to 2019. B) Publication rate of preprints on bioRxiv was steady around $70 \%$ until the end of 2017. Boxplot indicates median and quartiles of yearly distribution, points indicate monthly values. C) Numbers of published manuscripts from bioRxiv preprints parallels numbers of submitted preprints until early 2017. Points indicate monthly values. D) Combined violin- and boxplots show the distribution of publication intervals for any given year. The median interval from preprint to publication on bioRxiv remained steady across the measured period from 2013 to 2018. Starting 2019, the median predictably decreases due to the maximally possible duration of the interval for recent manuscripts (indicated by grey line). 


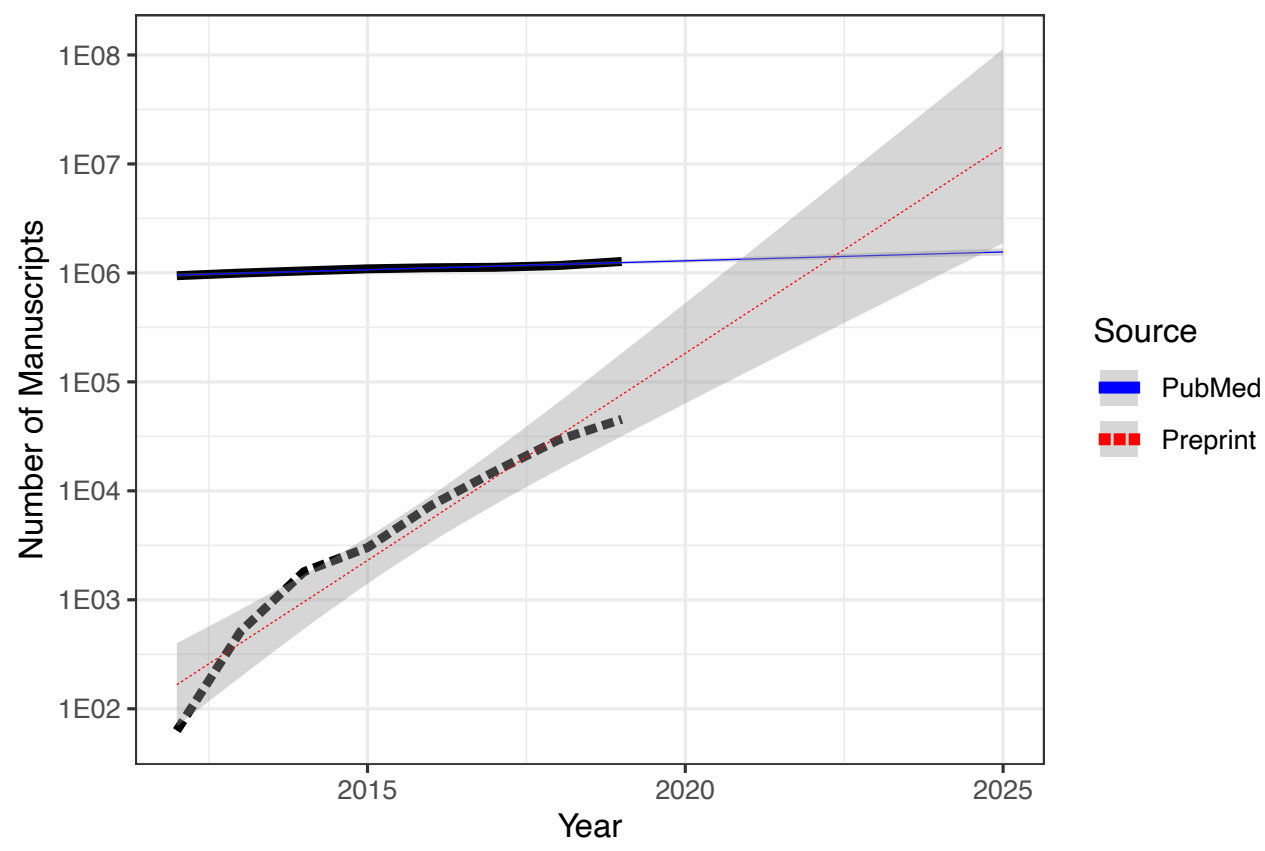

Supplementary Figure 1: Extrapolation of preprint and published manuscript numbers.

Linear regression based on the values between years 2012 and 2019 approximates the point of equal numbers of preprints and peer-reviewed manuscripts in the biomedical field around the year 2025 at the latest. Shaded area represents standard error. 